\title{
Migración de tránsito por la ruta del occidente de México: actores, riesgos y perfiles de vulnerabilidad
}

\author{
ADRIANA GONZÁLEZ ARIAS* \\ OLGA AIKIN ARALUCE**
}

RESUMEN: Este trabajo analiza las condiciones de vulnerabilidad que presentan distintos grupos de migrantes que transitan por la ruta del occidente de México. A partir de un concepto de vulnerabilidad centrado en los factores de riesgo y en las capacidades o recursos que los sujetos poseen para afrontarlos, se aplicaron entrevistas a 78 migrantes encontrados a lo largo de la ruta y se construyeron varias categorías de vulnerabilidad que nos permiten conocer las condiciones de tránsito. Encontramos que la vulnerabilidad de los migrantes entrevistados está fuertemente permeada por factores como la nacionalidad, el género, la edad, la experiencia previa del viaje, las redes de apoyo y la condición socioeconómica. En este sentido, los grupos menos vulnerables lo son básicamente por el factor de nacionalidad, en donde sólo un grupo de mexicanos y mexicanas muestran relativamente mayores recursos para afrontar los peligros (el tránsito legal o la existencia de redes de apoyo más sólidas).

PALABRAS CLAVE: migración de tránsito, ruta de occidente, migración, violaciones de derechos, seguridad.

* Profesora e investigadora del Instituto Tecnológico y de Estudios Superiores de Occidente, México.

*** Profesora e investigadora del Instituto Tecnológico y de Estudios Superiores de Occidente, México. 
ABSTRACT: This study analyzes the conditions of vulnerability exhibited by certain migrant groups that transit through the western route in Mexico. Derived from a concept of vulnerability based on risk factors and on the capabilities or resources that the enable the subjects to confront them, interviews were conducted with 78 migrants encountered along this route and which reside within various vulnerability categories that allow us to understand the conditions of transit. We find that the vulnerability of the interviewed migrants is strongly permeated by factors such as nationality, gender, age, previous migratory experience, networks of support and socioeconomic conditions. In this sense, the least vulnerable groups are those of a single nationality, in which a group of male and female Mexicans exhibits greater resources with which to confront dangers (legal transit or the existence of more reliable networks of support).

KEY WORDS: transit migration, western route, migration, violation of rights, security. 


\section{INTRODUCCIÓN*}

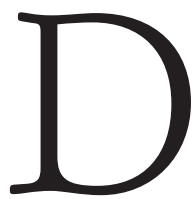

esde 2000, la migración de tránsito por el territorio mexicano con dirección a Estados Unidos ha sido denunciada a nivel nacional e internacional debido a la vulnerabilidad, riesgos y violaciones a los derechos humanos que padecen en ese recorrido los migrantes centroamericanos y mexicanos. Informes de la Comisión Nacional de Derechos Humanos (CNDH, 2009, 2011) señalan escalofriantes cifras sobre abusos a migrantes, donde destacan los secuestros y extorsiones. Otras organizaciones afirman que las autoridades migratorias o las fuerzas de seguridad mexicanas suelen participar directamente en estos abusos y que las agresiones se dan en un marco de total impunidad (Amnistía Internacional, 2010; Meyer y Brewer, 2010). Calificada como una «tragedia humanitaria» por actores internacionales, ${ }^{1}$ esta problemática cobró su punto más álgido en los medios internacionales a raíz de la matanza de 72 migrantes en San Fernando, Tamaulipas, en agosto de 2010, fecha en que fueron hallados los cuerpos. Para mayo de 2011, posiblemente vinculado a la denuncia y presión política, el gobierno federal publicaba la nueva Ley de Migración como un intento (malogrado para algunos) de humanizar la regulación de la migración en México. Frente a la política de criminalización del migrante y la militarización de la frontera sur como parte del entramado regional de contención migratoria, la nueva ley reconoce la necesidad de salvaguardar los derechos humanos de los migrantes y les otorga derechos puntuales en el campo de la salud, la educación, la sanidad y el registro civil.

\footnotetext{
* Agradecemos la colaboración de los estudiantes del ITeso, Alejandra Sandoval en la aplicación de entrevistas y sistematización de datos; Óscar Limón en la aplicación de entrevistas, y Gabriela Rangel en la sistematización de datos; así como a las voluntarias de la Iniciativa Kino Ashley Edgette y Natalia en la realización de entrevistas; Programa de Asuntos Migratorios de ITESO en contactos; FM4 Paso Libre en información y uso de instalaciones en Guadalajara; proyecto Iniciativa Kino para la Frontera en información y uso de instalaciones en Nogales; Daniel David González Arias en el diseño de mapas.

${ }^{1}$ Este es el calificativo que le dio la Comisión Interamericana a la referida situación durante la audiencia titulada "Situación de los derechos humanos de migrantes en tránsito por territorio mexicano» celebrada en marzo de 2010. Disponible en http://www.oas.org/es/cidh/ audiencias/TopicsList.aspx?Lang $=$ es\&Topic $=20$
} 
Si bien la migración de tránsito por el corredor mexicano ha cobrado un auge mediático reciente, la literatura académica viene haciendo mención de este fenómeno desde la década de los años ochenta, cuando los flujos procedentes de Centroamérica arribaban a México en busca del estatus de refugiados o con la intención de llegar a Estados Unidos para escapar de las atrocidades de las guerras civiles de la época, captaron la atención de las autoridades mexicanas y marcaron el rumbo de la política migratoria. A partir de 2000 y desde la óptica de la sociología, antropología, economía y ciencia política, la literatura académica analiza múltiples ángulos: perfil del migrante de tránsito y estrategias de supervivencia en el camino, redes sociales de apoyo, redes criminales que lo acechan, grupos de apoyo, discriminación o maltrato por ciudadanos mexicanos, violencia estructural de la que huyen, violaciones a los derechos humanos y política migratoria nacional o regional de los gobiernos involucrados para regular el fenómeno.

Entre los estudiosos, especial interés han suscitado las rutas de tránsito de los migrantes - principalmente de centroamericanos, aunque también mexicanos-y los peligros que enfrentan. Como soporte material del recorrido, las rutas son el punto de referencia para la actuación de los actores involucrados. Más allá del trazado genérico que encontramos en el mapa de Casillas y otros posteriores, poco sabemos de la ruta de occidente, que puede observarse de sur a norte del Océano Pacífico. Sin embargo, para efectos de esta investigación se tomará como ruta de occidente la que parte de Irapuato, Guanajuato, avanza por Jalisco, Nayarit y Sinaloa, y finaliza en Sonora o Baja California, siguiendo el trazado ferroviario. Si bien para la Zona Metropolitana de Guadalajara se publicaron dos diagnósticos acerca del perfil y características de los migrantes en tránsito (véase Rocha, Escalona y Gutiérrez, 2011; fm4 Paso Libre, 2013), no encontramos más datos de esta ruta en su totalidad. Por tanto, este trabajo intenta cubrir ese vacío y simultáneamente hace una contribución a la literatura académica sobre migración de tránsito por México.

El objetivo principal de esta investigación consiste en describir y analizar las condiciones de movilidad humana de los grupos migratorios que transitan por la ruta del occidente de México camino a la frontera norte. Para ello realizamos una descripción inicial de la ruta y analizamos a los acto-

$$
84 \frac{\text { PRIMER SEMESTRE } 2015}{\text { MIGRACIÓN Y DESARROLLO NÚM. } 24}
$$


res que por ella viajan, haciendo especial énfasis en los riesgos a la seguridad del migrante y en las capacidades de éstos para afrontarlos. A partir de los conceptos de riesgo y capacidades o recursos, logramos construir varias categorías de vulnerabilidad que nos ayudan a comprender las condiciones del viaje para los distintos grupos migratorios. Las preguntas que guiaron este proceso de búsqueda fueron: żquiénes transitan la ruta?, żqué riesgos los acechan y cómo los enfrentan?, żcuál es su capacidad de respuesta ante el peligro? y żquiénes tienen más posibilidades de éxito en este arriesgado viaje?

\section{CUADRO 1}

Descripción del grupo de estudio por lugar de entrevista

\begin{tabular}{lcccr}
\hline POR NACIONALIDAD & LUGAR DE LA ENTREVISTA & GUADALAJARA & NOGALES & TOTALES \\
\hline Mexicanos & Total & 4 & 9 & 13 \\
& Hombres & 3 & 5 & 8 \\
& Mujeres & 1 & 4 & 5 \\
Hondureños & Menores & - & 1 & 1 \\
& Total & 19 & 13 & 32 \\
& Hombres & 17 & 10 & 27 \\
\multirow{3}{*}{ Salvadoreñes } & Menores & 2 & 3 & 5 \\
& Total & 1 & 2 & 3 \\
& Hombres & 12 & 4 & 16 \\
Guatemaltecos & Mujeres & 12 & 4 & 16 \\
& Menores & - & - & - \\
& Total & 1 & 5 & 17 \\
Total & Hombres & 12 & 3 & 12 \\
& Mujeres & 9 & 2 & 5 \\
& Menores & 3 & - & 7 \\
\hline
\end{tabular}

Fuente: Elaboración propia con datos del trabajo de campo de esta investigación.

La investigación, básicamente de corte cualitativo, recupera fuentes documentales que arrojan luz sobre aspectos teóricos de interés, si bien se basa principalmente en una investigación de campo realizada entre enero y mayo de 2014. En ésta se aplicaron 78 entrevistas semiestructuradas a migrantes en tránsito hacia la frontera norte (46 realizadas en Guadalajara, Jalisco, y 32 en Nogales, Sonora) y se realizaron cinco entrevistas a informan- 
tes clave (investigadores y encargados de comedores para migrantes). En el cuadro 1 se describe el grupo de estudio. Los instrumentos aplicados fueron diseñados a partir de la técnica de etnografía multisituada.

\section{VULNERABILIDAD}

El concepto de vulnerabilidad ha incursionado con fuerza en los últimos 40 años en las ciencias sociales, específicamente en el campo de estudios del desarrollo. Según Pérez de Armiño (2009), dicho concepto constituye un "fértil instrumento de estudio de la realidad social, de disección de sus causas profundas, de análisis multidimensional que atiende no sólo a lo económico, sino también a los vínculos sociales, el peso político, el entorno físico y medioambiental o las relaciones de género, entre otros factores» (2009: 2). De ahí que sea un punto de partida importante para orientar las políticas públicas en cuestiones de desarrollo, así como las intervenciones de acción humanitaria.

Pérez de Armiño define la vulnerabilidad como:

El nivel de riesgo que afronta una familia o individuo a perder la vida, sus bienes y propiedades o su sistema de sustento, ante una posible catástrofe. Dicho nivel guarda también correspondencia con el grado de dificultad para recuperarse después de tal catástrofe, la indefensión o falta de medios para afrontar la situación sin pérdidas perjudiciales (Pérez de Armiño, 2009: 11).

De esta definición se desprenden dos componentes básicos: uno externo, compuesto por los riesgos o conjunto de amenazas potenciales y, otro interno, constituido por las capacidades de un individuo o grupo para afrontar dichos riesgos.

En una definición simplificada podemos decir que la vulnerabilidad es el nivel de exposición a riesgos y la dificultad para afrontarlos. ${ }^{2}$ Los riesgos

2 Un análisis más complejo de la vulnerabilidad incluye el conjunto de factores estructurales que condicionan y constriñen las capacidades del individuo y su posibilidad de respuesta, esto es, las causas profundas de la vulnerabilidad (Vogt, 2013; Pérez de Armiño, 2009). Para

$86 \frac{\text { PRIMER SEMESTRE } 2015}{\text { MIGRACIÓN Y DESARROLLO NÚM. } 24}$ 
están asociados a situaciones de inseguridad, incertidumbre y desprotección, las cuales constituyen un conjunto de amenazas potenciales o reales. La capacidad de defensa/respuesta o situación de indefensión propia de la vulnerabilidad deriva de la carencia de recursos del individuo dadas sus características personales (edad, sexo, escolaridad, condición étnica, situación migratoria o condición socioeconómica) y otros factores estructurales. Todo ello condiciona las posibilidades de acción y estrategia del individuo o grupo. Según Kazman (citado en Ibarra, 2009: 206), la capacidad de respuesta depende de los activos disponibles (como conjunto de recursos materiales e inmateriales sobre los que se posee control) y de los mecanismos de apoyo externo a los que se tiene acceso. Parte de estos activos también lo constituyen las habilidades adaptativas o estrategias de afrontamiento que implementan los actores.

El concepto de vulnerabilidad que aquí presentamos incorpora la noción de agencia, la idea del actor social, en donde se atribuye que el migrante, más allá de ser una víctima o sujeto pasivo constreñido por las condiciones estructurales adversas, posee «la capacidad para procesar la experiencia social y diseñar maneras de lidiar con la vida, aún bajo las formas más extremas de coerción» (Long, citado en Rivas, 2011: 17).

La vulnerabilidad como dificultad para resistir riesgos, o la capacidad/ incapacidad para reponerse después de que éstos se hayan materializado, depende de diversos factores, como la edad, el nivel de salud, la escolaridad, la situación socioeconómica o el capital social que posee el sujeto. Depende del conjunto de características personales del individuo, los activos que posea, los apoyos que recibe y las estrategias que es capaz de implementar.

Pérez de Armiño la vulnerabilidad «responde a una combinación de múltiples factores geográficos, económicos, sociales, políticos y personales». Sus causas son de tres tipos: causas raíces o estructurales, los procesos de crisis a medio o corto plazo y los determinantes personales, constituyéndose de esta forma "toda una cadena explicativa que va de lo macro» y estructural a lo «micro; desde las relaciones sociales globales hasta las condiciones específicas de cada individuo» (2009: 13). Si bien la definición de vulnerabilidad propuesta en este artículo está, por cuestiones operativas, básicamente centrada en el nivel individual, no ignora la importancia que reviste contemplar los constreñimientos estructurales y los procesos de crisis en los lugares de origen y tránsito de los grupos migratorios estudiados. 


\section{CAPACIDADES = CARACTERÍSTICAS PERSONALES + ACTIVOS (CAPITAL SOCIAL + APOYOS + HABILIDADES ADAPTATIVAS)}

Desde la perspectiva del actor social resulta especialmente interesante el concepto de capital social como activo fundamental para enfrentar situaciones de vulnerabilidad. Este concepto, importante dentro de la teoría de redes, remite al poder de agencia de los migrantes y sus comunidades a la hora de organizar los procesos de migración e incorporación en lugares de destino. El capital social, como lazos familiares, amistosos y de la comunidad, proveen al migrante de un gran apoyo en el proceso migratorio, incrementando las posibilidades migratorias y disminuyendo los costos o riesgos del desplazamiento. Estas redes que forman el capital social representan «mecanismos de transmisión de capital cultural» que proveen información sobre las oportunidades migratorias, las rutas y las posibles estrategias (Castles, 2010).

Con ánimo de lograr indicadores que nos ayudarán a dimensionar la vulnerabilidad en sus distintos aspectos, construimos la siguiente matriz:

\section{CUADRO 2}

Perfil de vulnerabilidad $=$ riesgos + capacidades

\begin{tabular}{|c|c|c|c|c|}
\hline \multicolumn{3}{|c|}{ RIESGOS DURANTE LA RUTA } & \multicolumn{2}{|c|}{ CAPACIDADES } \\
\hline NATURALES & $\begin{array}{c}\text { INSEGURIDAD } \\
\text { PÚBLICA }\end{array}$ & INSTITUCIONALES & CONDICIONES PERSONALES & ACTIVOS \\
\hline $\begin{array}{l}\text { Climáticos. } \\
\text { Propios del } \\
\text { medio de } \\
\text { transporte } \\
\text { (tren, autobús, } \\
\text { etc.). }\end{array}$ & $\begin{array}{l}\text { Hechos delicti- } \\
\text { vos cometidos } \\
\text { por distintos } \\
\text { grupos de } \\
\text { actores. }\end{array}$ & $\begin{array}{l}\text { Control migratorio } \\
\text { (retenes, operativos, } \\
\text { estaciones migra- } \\
\text { torias, etc.). }\end{array}$ & $\begin{array}{l}\text { Características físicas } \\
\text { y de salud. } \\
\text { Perfil socioeconómico: } \\
\text { Nacionalidad, } \\
\text { Sexo, } \\
\text { Edad, } \\
\text { Escolaridad, } \\
\text { Oficio. } \\
\text { Estatus jurídico: } \\
\text { Identificación personal } \\
\text { Documentación } \\
\text { migratoria. }\end{array}$ & $\begin{array}{l}\text { Capital social } \\
\text { (familia, redes de } \\
\text { apoyo, redes } \\
\text { sociales, acceso a } \\
\text { información). } \\
\text { Habilidades } \\
\text { adaptativas o } \\
\text { estrategias. } \\
\text { Apoyos externos. }\end{array}$ \\
\hline
\end{tabular}

Fuente: Elaboración propia a partir del cuaderno Seguridad para el Migrante: una agenda por construir de INEDIM (2011).

$88 \frac{\text { PRIMER SEMESTRE } 2015}{\text { MIGRACIÓN Y DESARROLLO NÚM. } 24}$ 


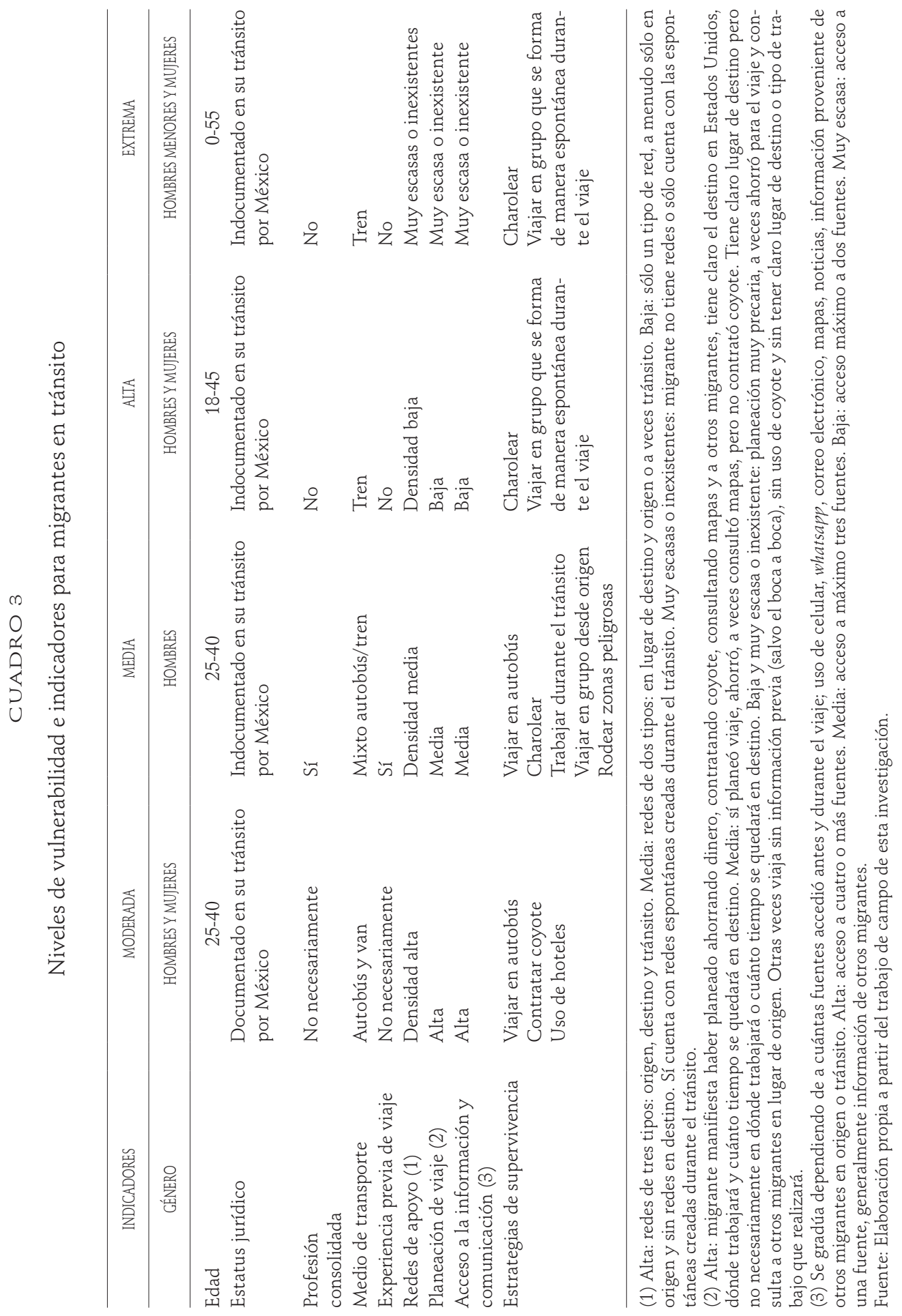


Los riesgos responden a tres tipos de categorías: naturales (condiciones climáticas y riesgos inherentes al tipo de transporte), inseguridad pública (incluyen la tipología de hechos delictivos a los que están expuestos u otros tipos de violencia social que los acechan) e institucionales (los obstáculos y violaciones a los derechos humanos que derivan del control migratorio). Asimismo, las capacidades las dividimos en las condiciones personales del migrante (perfil socioeconómico y estatus migratorio) y activos disponibles (básicamente el capital social, habilidades adaptativas o estrategias y apoyos externos que encuentra por la ruta). A partir de estos elementos construimos una entrevista semiestructurada que aplicamos a 78 migrantes que viajaban por la ruta. El grupo de estudio, si bien no tiene un valor estadístico, nos permitió llegar a un efecto de «saturación», donde al repetirse ciertas características y patrones, logramos establecer cuatro perfiles de vulnerabilidad básicos. Estos perfiles responden a ciertos indicadores relacionados con género, edad, estatus jurídico, profesión, experiencia previa de viaje, redes de apoyo, planeación de viaje y acceso a la información. El siguiente cuadro expone los indicadores a los que responde cada categoría de vulnerabilidad.

En los siguientes tres apartados presentamos los resultados de la investigación. Iniciamos con una descripción de la ruta y los riesgos inherentes a la misma, en una segunda sección categorizamos a los grupos en tránsito a partir de sus condiciones personales y capacidad de respuesta ante los peligros.

\section{RUTA DE OCCIDENTE: UNA DESCRIPCIÓN INICIAL}

La ruta del occidente de México tiene una historia que se puede remontar a más de 100 años, ya que enlaza el norte con estados tradicionalmente expulsores de migrantes como Guanajuato, Michoacán, Jalisco y Zacatecas (Machuca, 2014). No obstante, su visibilidad mediática acontece en los últimos años, notablemente a partir de 2010, cuando los migrantes cambian el itinerario por el incremento de la violencia en las rutas del este de México. Entre 2008 y 2014, fuentes de información mexicanas observan un incremento en los flujos de migrantes mexicanos y centroamericanos que pasan por el occi-

$90 \frac{\text { PRIMER SEMESTRE } 2015}{\text { MIGRACIÓN Y DESARROLLO NÚM. } 24}$ 
dente de México. ${ }^{3}$ La ruta de occidente, si bien fue históricamente la más transitada en el territorio mexicano, en los últimos años ha presentado un flujo migratorio descendente en relación con las otras rutas. De acuerdo con las detenciones realizadas por la Patrulla Fronteriza de Estados Unidos entre 2000 y 2014 (Southwest Border Sectors, 2014), se registró un decrecimiento de 86 por ciento en las detenciones operadas en San Diego y Tucson, mientras que en los puntos fronterizos de la ruta del Golfo éstas se incrementaron, entre 2011 y 2014, en 326 por ciento. Asimismo, las cifras de la Patrulla Fronteriza arrojan que el flujo de centroamericanos se ha incrementado en general, siendo que del total de aprehendidos en la frontera en 2014, 53 por ciento fueron centroamericanos, lo que contrasta con las cifras de años anteriores, en donde entre 2000 y 2009, el grupo de centroamericanos sólo representaba 6 por ciento de los detenidos. Si bien históricamente el tránsito por el occidente ha descendido comparativamente en relación con otras rutas, en términos absolutos ha incrementado en los últimos años, lo que parcialmente podría explicarse por el aumento del flujo de centroamericanos que transitan por todo México, así como por la intensa violencia asociada a las rutas del este del país.

Grosso modo, de acuerdo con los lugares de viaje que obtuvimos en las entrevistas, podemos decir que el trazado de la ruta coincide con la línea ferroviaria que va a Estados Unidos, iniciando en Irapuato, Guanajuato, y concluyendo en diversos puntos de la frontera occidental. Si observamos el mapa 1, vemos que el camino es básicamente unidireccional desde Irapuato hasta Santa Ana, Sonora, donde se bifurca en dos direcciones: a Nogales y a

${ }^{3}$ El aumento de mexicanos se observa en la muestra de la Encuesta sobre Migración en las Fronteras Norte y Sur de México (Emif-Norte, 2011: 259), en ésta los migrantes que llegaron a alguna ciudad fronteriza del oeste del país en los últimos 5 años reportados representan, con respecto al total de los levantamientos de las zonas centro y este de México, 13.3 por ciento en 2007, 31.52 por ciento en 2008, 27.4 por ciento en 2009, 45 por ciento en 2010 y 41.9 por ciento en 2011. En lo que corresponde a los centroamericanos, los boletines estadísticos de la Unidad de Política Migratoria de la Secretaría de Gobernación (Segob) señalan un aumento en las detenciones en los estados que corresponden a la ruta de occidente (Baja California, Guanajuato, Jalisco, Nayarit, Sinaloa y Sonora), en donde en 2011 hubo 3,434 centroamericanos detenidos; 3,962 en 2012; 3,413 en 2013, y 4,298 en 2014 (http://www. gobernacion.gob.mx/es_mx/SEGOB/Boletines_Estadisticos, revisados en enero de 2015). 
Mexicali. Muchos de los migrantes que optan por viajar hacia Mexicali deciden, un poco más adelante, en Caborca, bajarse del tren y llegar caminando o en autobús a Altar, Sonora, donde esperan unos días para buscar un coyote que los lleve a Sásabe, punto de cruce muy recurrido. Otros se dirigen hacia Sonoyta, Sonora, buscando un cruce solitario o asistido, mientras que otros se van a Mexicali. Casi todos buscan cruzar la frontera, si bien muchos lo retrasan para trabajar y ahorrar el dinero suficiente para pagar a coyotes y mafias. Otros, ante el fracaso del cruce, se quedan en las zonas fronterizas, también buscando trabajo y nuevas oportunidades de paso.

\section{Mapa 1}

Ruta de tránsito migratorio por el occidente de México

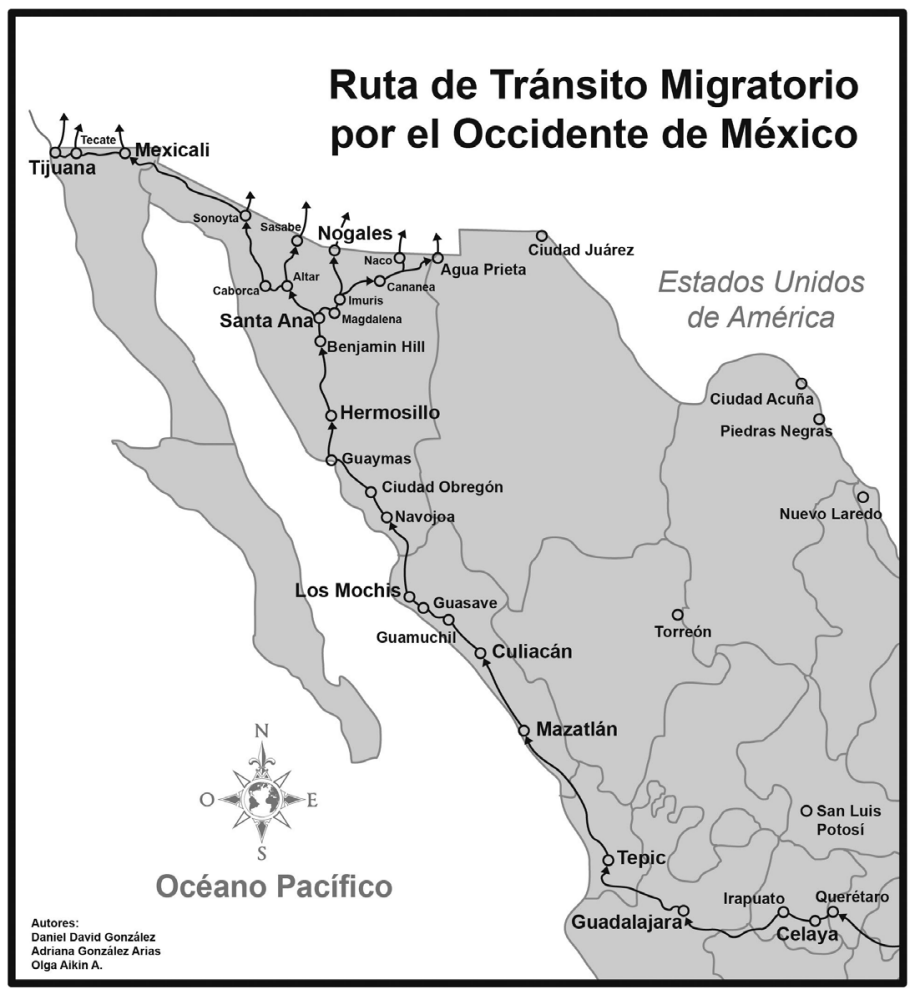

Fuente: Elaboración propia con datos del trabajo de campo de esta investigación.

$92 \frac{\text { PRIMER SEMESTRE } 2015}{\text { MIGRACIÓN Y DESARROLLO NÚM. } 24}$ 


\section{Perfil general de los migrantes}

Nuestra investigación de campo se realizó en Guadalajara (en el comedor de FM4) y Nogales (en el comedor de la Iniciativa Kino) y se enfocó a estudiar los flujos migratorios hacia el norte, con intención de llegar a la frontera o ya una vez en ella. En las 78 entrevistas encontramos cuatro grandes grupos nacionales que transitan la ruta hacia el norte: mexicanos, hondureños, salvadoreños y guatemaltecos. Buscamos obtener un grupo mínimamente representativo de todas las nacionalidades encontradas, si bien el grupo de entrevistados más numeroso fue de hondureños (41.7 por ciento) seguido de guatemaltecos (21.5 por ciento), salvadoreños (20.2 por ciento) y mexicanos (16.4 por ciento). ${ }^{4}$ En el grupo de estudio predominaron los hombres adultos (80.7 por ciento frente a 19.23 por ciento de mujeres) con un rango de edad de entre 20 y 29 años (40.7 por ciento), grupo de edad que predominó frente a los menores (8 por ciento) o mayores de 50 años (5 por ciento).

Los migrantes entrevistados siguieron este trazado principal viajando, ya fuera exclusivamente en tren (41 por ciento), en autobús (12.8 por ciento) o mezclando ambos transportes (44.8 por ciento), y pasaron por las ciudades principales de la ruta. La racionalidad que permea esta decisión responde, según Chávez y Landa (2012), a factores de nacionalidad y de clase socioeconómica. Mientras que los grupos más pobres suelen viajar en tren (un medio más peligroso, pero que esquiva los controles migratorios), los que tienen más recursos o una situación de tránsito regular (como los mexicanos) tienden a tomar el autobús, el cual proporciona mayor seguridad pero supone más costo monetario por el pago de transporte y las constantes extorsiones de las autoridades. En nuestro grupo de estudio predominó la decisión de combinar ambos medios de transporte, una estrategia recurrente que rompe con

${ }^{4}$ Estos porcentajes no reflejan cuál de los grupos es el más numeroso, ya que no era el objetivo de la muestra y existe esa información en otros estudios. Por ejemplo, el reporte de FM4 (2013) estima que el grupo en tránsito más numeroso por la Zona Metropolitana de Guadalajara es el de los hondureños (que representan 43 por ciento), seguido del grupo de mexicanos (30.2 por ciento), los guatemaltecos (9.6 por ciento), salvadoreños ( 9.6 por ciento) y nicaragüenses (1.5 por ciento). No obstante, en nuestra investigación de campo no encontramos nicaragüenses para entrevistar. 
el criterio de condición económica y en la que predomina la búsqueda de seguridad como condición prioritaria.

Gran parte de los entrevistados respondían al perfil del migrante por razones económicas (76.9 por ciento) y casi 9 por ciento manifestó haber salido de su país por cuestiones de violencia (básicamente la agresión propia de un ambiente social permeado por el narcotráfico y el pandillerismo). No obstante, para 30.7 por ciento la mezcla de ambos motivos (económicos y de violencia) fueron la causa determinante de la migración. En este sentido, es importante señalar que la pobreza es el motor principal de expulsión de esta población, variable que a menudo se conjunta y refuerza con el factor violencia.

47.43 por ciento de los entrevistados viajaba a Estados Unidos por primera vez y casi la otra mitad (47.33 por ciento) ya habían realizado el viaje una o varias veces. Esto corresponde con otro dato significativo: 52 por ciento había sido deportado de Estados Unidos y buscaba regresar.

Dentro del grupo de mexicanos encontramos muchos deportados que albergaban la intención de volver a Estados Unidos y también algunos jornaleros en busca de trabajos temporales en lugares como Nayarit, Baja California, Sinaloa o Sonora. En este sentido, la ruta es transitada en ambas direcciones. Sin embargo, la mayoría de los centroamericanos, deportados o no, se dirigían a la frontera norte con la intención de cruzarla lo más pronto posible. Una parte considerable de los entrevistados se dirigían a California (31 por ciento), mientras que otros destinos de relevancia eran Arizona (10 por ciento), Texas (10 por ciento) y Nuevo México (10 por ciento). Estos datos podrían indicar que en la racionalidad de la elección de la ruta, además de la búsqueda de seguridad, también influyen factores como el destino final del viaje.

Si bien nuestro trabajo se centró en observar el flujo «hacia arriba», no obstante esta ruta también es transitada "hacia abajo», incluso lateralmente; los flujos son complejos y circulan en distintas direcciones. Es más, el flujo circula, pero también se estanca. Así lo señala Iliana Martínez, investigadora del Programa de Asuntos Migratorios (Prami) del Instituto Tecnológico y de Estudios Superiores de Occidente (ITESO), quien estudia grupos de deportados que se estancan en alguna población del Bajío, o circulan acotadamente por sub-rutas, si bien siguen albergando la intención de cruzar de nueva cuenta. La ruta, en este sentido, presenta un fenómeno de movilidad humana complejo, 
difícil de cuantificar y comprender, frecuentemente permeado por el fracaso migratorio y en el que el movimiento se da en varias direcciones. El viaje y el tránsito se prolongan y la zona del Bajío a menudo funciona como un oasis donde los migrantes pueden estar temporadas largas, moverse por las sub-rutas del corredor y sobrevivir (Martínez, 2014).

El fenómeno de estancamiento o tapón también se presenta en la zona fronteriza y está relacionado con el fracaso migratorio y las políticas de contención implementadas por Estados Unidos. En este sentido, las entrevistas arrojan muchos casos de personas cuyo viaje se alarga ante los múltiples obstáculos (institucionales y criminales) y que esperan reunir los recursos necesarios para cruzar por primera o sucesivas veces.

\section{El mapa del peligro}

Según el reporte de Foote y Small (2013), Inseguridad permanente: abusos contra centroamericanos en México, en la ruta de occidente el crimen organizado está menos presente, pero la extorsión de la policía es muy elevada y las condiciones climáticas de la ruta son más peligrosas que otras. Esta afirmación coincide con nuestros hallazgos, en donde el problema más recurrente reportado por los migrantes fue el abuso de las autoridades ${ }^{5}$ (policías federales y locales, aunque también agentes de migración que extorsionaron a los migrantes). 59 por ciento de los entrevistados manifiesta haber tenido este tipo de problema, señalando especialmente lugares como Celaya e Irapuato, Guanajuato, y Mazatlán, Sinaloa.

No obstante, 37 por ciento de los entrevistados afirmó haber sido agredido por el crimen organizado, mafias y grupos pandilleros durante el tránsito por México, si bien casi 73 por ciento de estas agresiones ocurrieron en

${ }^{5}$ En el análisis de datos no fue posible separar las agresiones de policías en relación con las que se dieron por agentes de migración. Esto debido a que los migrantes no saben identificar con claridad quién pertenece a qué institución, los mencionan en la mayoría de las veces de manera generalizada. 
CUADRO 4

Problemas y abusos experimentados por los migrantes en la ruta del Pacífico

\begin{tabular}{lc}
\hline \multicolumn{1}{c}{$\begin{array}{c}\text { TIPOS DE PROBLEMAS/ABUSOS } \\
\text { DURANTE EL VIAJE }\end{array}$} & $\begin{array}{c}\text { \% DE ENTREVISTADOS QUE SUFRIERON } \\
\text { ESTOS PROBLEMAS/ABUSOS }\end{array}$ \\
\hline Extorsión de policías y agentes de migración & 59.0 \\
Agresión de grupos criminales (pandilleros, mafias y & 37.0 \\
narcotraficantes) & 32.0 \\
Agresión de otros ciudadanos mexicanos & 20.5 \\
Accidentes en el tren o por el camino & 69.0 \\
Problemas de salud & \\
\hline
\end{tabular}

Fuente: Elaboración propia con datos del trabajo de campo de esta investigación.

la parte sur y centro del país. 10 por ciento señala como zonas «calientes» a Sinaloa (específicamente Mazatlán, por la presencia de pandillas) y Sonora (específicamente Altar, Nogales y otros puntos de la frontera debido a la presencia del crimen organizado y mafias). Estos datos no nos permiten afirmar, de forma contundente, que la ruta del occidente es la más segura, o cuantificar puntualmente la incidencia de la agresión de los distintos grupos criminales. No obstante, la mayoría de los migrantes afirma que optaron por esta ruta por presentar condiciones más seguras: concretamente menos presencia del crimen organizado y de operativos migratorios. Si bien ésta parece ser la percepción que predomina entre ellos, las condiciones de la ruta podrían agravarse en un futuro si tenemos en cuenta que, en la medida en que los migrantes ajustan los itinerarios del viaje en búsqueda de mayor seguridad, sus victimarios también lo hacen, por lo que el abuso tiende a perseguirlos (Casillas, 2008; Foote y Small, 2013).

Si bien las extorsiones de autoridades y las agresiones de grupos criminales presentan la fuente de mayor preocupación de los migrantes, no obstante 32 por ciento afirma haber sido agredido por otros ciudadanos mexicanos mediante insultos, robos, extorsiones y otras agresiones. Los eventos más frecuentes se produjeron con trabajadores de la línea ferroviaria más que con ciudadanos de a pie.

$96 \frac{\text { PRIMER SEMESTRE } 2015}{\text { MIGRACIÓN Y DESARROLLO NÚM. } 24}$ 


\section{CUADRO 5}

Agresiones del crimen organizado, las mafias y pandilleros contra migrantes por estados en las que ocurrieron

\begin{tabular}{lcc}
\hline \multicolumn{1}{c}{ ESTADO } & $\begin{array}{c}\text { NÚMERO DE } \\
\text { AGRESIONES } \\
\text { (EVENTOS) }\end{array}$ & $\begin{array}{c}\text { VALOR } \\
\text { PORCENTUAL }\end{array}$ \\
\hline Veracruz & 19 & 39.58 \\
Estado de México & 8 & 16.6 \\
Chiapas & 5 & 10.4 \\
Sinaloa & 5 & 10.4 \\
Sonora & 5 & 10.4 \\
Tabasco & 3 & 6.25 \\
Guanajuato & 1 & 2.08 \\
Jalisco & 1 & 2.08 \\
Nayarit & 1 & 2.08 \\
Total: & 48 & 100 \\
\hline
\end{tabular}

Fuente: Elaboración propia con datos del trabajo de campo de esta investigación.

Los accidentes de tren o durante el camino representan un cuarto grupo de problemas que enfrentan los migrantes durante su tránsito por este corredor: 20.5 por ciento manifiesta haberlos sufrido. Asimismo, 69 por ciento presentó problemas de salud durante el viaje, condición que podríamos explicar como consecuencia, en gran medida, de las agresiones y problemas anteriormente citados.

\section{FLUJOS MIGRATORIOS Y VULNERABILIDAD: UN INTENTO DE CATEGORIZACIÓN}

Los migrantes encontrados en nuestro trabajo de campo constituyen un universo variopinto de nacionalidades, edades, itinerarios, motivaciones, condiciones socioeconómicas y recursos. Más allá de lo difícil que resulta describir, con un mínimo de fidelidad, una realidad tan diversa y compleja, o las limitaciones que tenemos los científicos sociales para encajar la realidad en categorías construidas, pretendemos encontrar patrones comunes que 
nos permitan hacer ciertas generalizaciones. Con las limitaciones señaladas, a partir de la observación de ciertas regularidades en las entrevistas, construimos cuatro categorías básicas de vulnerabilidad a las que esta población aparentemente respondía. Encontramos que casi 70 por ciento del grupo de estudio se ubica dentro de cuatro tipos de vulnerabilidad: moderada, media, alta y extrema, si bien 32 por ciento difícilmente encaja en éstos y presenta indicadores en varios de ellos. No obstante, este porcentaje quedó incluido en alguna de las modalidades de vulnerabilidad. La sistematización y clasificación de los datos, de acuerdo con nuestros indicadores, arroja que gran parte de la población encuestada se ubica en los rangos medios de vulnerabilidad (80 por ciento responde a las características de las categorías media y alta), mientras que un porcentaje menor (18.4 por ciento) encaja en las categorías de los extremos (vulnerabilidad moderada y vulnerabilidad extrema) presentando la característica forma acampanada de la curva de Bell.

\section{CUADRO 6}

Categorías de vulnerabilidad del grupo de estudio en porcentajes

\begin{tabular}{lcccc}
\hline & $\begin{array}{c}\text { VULNERABILIDAD } \\
\text { MODERADA }\end{array}$ & $\begin{array}{c}\text { VULNERABILIDAD } \\
\text { MEDIA }\end{array}$ & $\begin{array}{c}\text { VULNERABILIDAD } \\
\text { ALTA }\end{array}$ & $\begin{array}{c}\text { VULNERABILIDAD } \\
\text { EXTREMA }\end{array}$ \\
\hline Total de la muestra en porcentajes & 9.2 & 46 & 35.5 & 9.2 \\
\hline
\end{tabular}

Fuente: Elaboración propia con datos del trabajo de campo de esta investigación.

Perfiles predominantes y categorías de vulnerabilidad

Si bien pudimos apreciar que el viaje y sus peligros son muy elevados para una población en circunstancias precarias, no obstante se observa que el nivel de vulnerabilidad varía de acuerdo con factores como nacionalidad, experiencia previa de viaje, género y edad. A continuación describimos las categorías de vulnerabilidad encontradas y los perfiles de migrantes que predominan en ellas.

$98 \frac{\text { PRIMER SEMESTRE } 2015}{\text { MIGRACIÓN Y DESARROLLO NÚM. } 24}$ 
Vulnerabilidad moderada: la nacionalidad ayuda

Un primer grupo detectado es el que llamamos de vulnerabilidad moderada, en el cual se ubican hombres o mujeres mexicanos que viajan a Estados Unidos de forma documentada y cuyos activos más importantes consisten en poseer la nacionalidad mexicana y densas redes de apoyo en Estados Unidos, su propio país y durante el mismo viaje. Cuentan con varios familiares en Estados Unidos que les proporcionan recursos económicos y logísticos para pagar un coyote, organizar el viaje de la manera menos riesgosa posible y ser recibidos en el lugar de destino, si es que logran llegar. Al viajar de forma documentada por su propio país no enfrentan el riesgo de la deportación, lo que les permite un tránsito más cómodo, casi siempre en autobús, camionetas vans y a veces en avión, incluso algunos pueden alojarse en hoteles en ciertos puntos críticos. Suelen viajar en grupo desde el inicio del viaje, y si bien en este grupo encontramos menores y mujeres, los factores edad, experiencia previa o género no resultan tan determinantes, dado el nivel de protección con el que viajan. Aunque estas condiciones permiten mantener los riesgos relativamente bajos durante gran parte del viaje, el riesgo del «cruce» sigue siendo elevado y no están exentos de extorsiones y abusos de la policía o mafias locales en ciertos puntos del viaje.

Este grupo utiliza estrategias de seguridad efectivas (forma de transporte más seguro, uso de coyote u hospedaje en hoteles) y logra mantener buena comunicación con sus redes de apoyo mediante el uso de teléfono celular, facebook o aplicaciones de mensajes instantáneos como el whatsapp. Para el cruce de la frontera el riesgo es muy alto, aunque cuentan con mayores recursos para afrontar el peligro. Si son deportados podrán esperar en la frontera a que sus familiares les subvencionen y organicen un segundo cruce.

Un caso que ilustra bien esta categoría es el grupo de cuatro mexicanos entrevistados procedentes de Zitácuaro, Michoacán, formado por una pareja de 40 y 42 años (Aurelio y Verónica) que viajaban con dos vecinos de 18 y 23 años (Brianda y Gustavo). Aurelio y Verónica vivieron 11 años en Estados Unidos (Anaheim, California) y salieron del país para visitar a su familia en México y ahora intentan volver. Son campesinos y tienen hermanos e hijos 
en California, los cuales les mandan dinero para el viaje y les consiguieron coyote desde el inicio del viaje. Después de vivir tantos años en Estados Unidos tienen amigos y familiares que los apoyan para hacer el viaje con consejos, dinero, logística o recargándoles el teléfono. Brianda y Gustavo también tienen vínculos importantes en California (padres y hermanos) que les patrocinan el viaje, si bien es la primera vez que lo hacen. Inclusive el equipo de basquetbol de Brianda, en Michoacán, se organizó para recaudar dinero y le dieron algo para los gastos. Lograron reunir 8,500 pesos cada uno y salieron de Zitácuaro con coyote, dirigiéndose en autobús hasta Nogales. Algunas noches dormían en hotel y como grupo compacto se apoyaban entre todos. Además del uso de celular, también durante el camino accedieron a facebook y correo electrónico para mantener una buena comunicación con sus redes. Junto con sus contactos en Estados Unidos planearon con cuidado el viaje para evitar algunos peligros. Todos tienen claro qué quieren hacer en la vida. Aurelio quiere estar 10 años más en Estados Unidos, reunir dinero y retirarse en Michoacán junto con su esposa para disfrutar su casa y familia. Brianda planea trabajar un año en Estados Unidos (con un familiar que dirige una fábrica), ahorrar y poder pagar su título de licenciada en contabilidad para posteriormente poner su propio despacho en su lugar de origen.

A pesar del perfil de vulnerabilidad moderada en el que inicialmente caen, el cruce está lleno de peligros y riesgos a veces letales. El coyote intentó hacer el cruce rodeando el muro de Nogales, Sonora, adentrándose en el cerro. Alegaba que era más seguro y rápido. Les ahorró el trato con la mafia local del cerro. Sin embargo, una vez pasada la «línea», los abandonó, perdieron la suma pagada (6,000 pesos cada uno). Verónica se lastimó el pie y, en esa situación, tuvieron que deambular por lugares inhóspitos y dormir a la intemperie dos días. Finalmente, la Patrulla Fronteriza los rescató y deportó. Ahora están de nuevo en Nogales esperando a que su familia ahorre y pueda volver a mandarles dinero para intentar un segundo cruce.

Vulnerabilidad media o la ventaja del deportado

Para aquellos que no cuentan con la nacionalidad mexicana, un activo socorrido es haber hecho el viaje previamente, contar con un oficio consoli-

$100 \frac{\text { PRIMER SEMESTRE } 2015}{\text { MIGRACIÓN Y DESARROLLO NÚM. } 24}$ 
dado y haber vivido en Estados Unidos. En este grupo se ubican, mayoritariamente, hombres centroamericanos, entre 25 y 40 años, de escasos recursos y que fueron deportados de Estados Unidos. Al haber vivido y trabajado cierto tiempo en ese país, aunque de forma indocumentada, adquirieron buena experiencia laboral como carpinteros, obreros de fábrica, jornaleros, etcétera, y lograron crear ciertas redes de apoyo (amigos, familia, patrones), fraguando una vida súbitamente interrumpida que ahora desean retomar a cualquier precio. A menudo no tienen grandes redes de apoyo en su país de origen (aunque sí familiares de muy escasos recursos que pueden depender de ellos), pero sí tienen algunas en Estados Unidos que les apoyan económica y logísticamente (aunque de forma modesta) para realizar el viaje o al menos para recibirlos cuando lleguen. El tener un oficio - a menudo consolidado en un mercado laboral competitivo como el de Estados Unidos- les permite sobrevivir el viaje y lograr ganar cierto dinero, en tránsito o destino, a través de su trabajo. Fruto de su experiencia previa de tránsito por México, poseen información y son capaces de implementar múltiples estrategias de sobrevivencia. Sus posibilidades de llegar al destino final son menores que las del grupo de vulnerabilidad moderada, pero superiores a los grupos de alta y extrema.

A menudo viajan solos y durante el camino forman grupos de apoyo que les proporcionan información y protección durante el viaje. Son vínculos espontáneos y frágiles (perentorios) pero de gran ayuda en el terreno. Viajan indocumentados por México, predominantemente en tren. Deciden esquivar los tramos más peligrosos — como Mazatlán, Orizaba y otros lugares del sur - tomando autobús o a pie. Al tener menos recursos que la categoría anterior, no suelen viajar con coyote por México, si bien algunos esperan poder contratar uno para el cruce de frontera, donde muchos dicen que trabajarán para poder pagarlo. Conocen el camino y sus riesgos, ante lo cual implementan un repertorio considerable de estrategias de supervivencia: ahorran algo para el viaje y calculan que sus mayores gastos serán en agua, comida y pago de extorsiones (policía, mafia...). En cierto punto del viaje se quedan sin dinero y a menudo charolean en puntos urbanos seguros o consiguen trabajos precarios para cubrir las necesidades básicas. El tren es un transporte 
conveniente porque ahí no hay operativos del Instituto Nacional de Migración (INM) y, por lo tanto, riesgo de deportación, pese a que las cuotas de las mafias y los robos del crimen organizado son frecuentes. No llevan mucho dinero encima porque saben que se lo robarán, de tener algunos recursos monetarios prefieren que sus familiares (de contar con su apoyo) se lo manden en puntos concretos del camino. Saben que el tren es muy peligroso y tienen estrategias precisas para no accidentarse, como no subirse cuando pasa deprisa, amarrarse siempre, meterse dentro de las góndolas para aguantar las pedradas de los «cholos» en su paso por Mazatlán e incluso reunir piedras para defenderse de los «bajadores». No llevan celular (porque se lo roban) pero suelen mantener contacto con sus redes mediante el uso de teléfonos públicos y, ocasionalmente, por facebook o correo electrónico en cibercafés o casas de migrantes. Aunque consultan mapas en las casas de migrantes, u ocasionalmente se ilustran a través de las noticias de los periódicos, la mayor fuente de información sobre la ruta y los peligros es su propia experiencia o la de otros migrantes. Por lo anterior, deducimos que el acceso a la información es escaso y que, salvo los vínculos espontáneos que forman en el trayecto, suelen estar solos.

Un caso que ilustra bien este tipo es el de José Santos, original de Varsovia, Honduras, un agricultor de 33 años. Vivió en Estados Unidos dos años y medio y ha sido deportado varias veces. Esta es la cuarta vez que realiza el viaje por la ruta del Pacífico. Tiene dos hermanos en Estados Unidos y otros amigos que le dan un poco de dinero para el viaje y lo recibirán cuando llegue. Viaja en tren por falta de recursos y para evitar a la «migra», pero el tramo de Orizaba a Lechería decidió esquivarlo tomando un autobús. En ciertos lugares charolea para obtener la comida diaria. Fruto de la experiencia acumulada, tiene muchos consejos que dar: «no confiar en nadie, no tomar alcohol, llevar poco dinero, mejor que te lo manden, amarrarse al tren aunque vayas despierto, sólo dormirse si te metes en una góndola, no acercarse a las ruedas». Para el paso por Mazatlán, advierte: «mucho cuidado, tiran piedras e intentan treparse al tren, son grupos de unos 10, chamacos de 15 a 17 años. Hay que buscar vagones que estén cubiertos para aguantar las pedradas. Si la mafia

$102 \frac{\text { PRIMER SEMESTRE } 2015}{\text { MIGRACIÓN Y DESARROLLO NÚM. } 24}$ 
logra subir y te atraca, dales todo lo que llevas para que no te agredan. Mejor ir solo, ellos buscan al grupo».

Si bien José Santos no tiene dinero para pagar coyote en ningún tramo del viaje, su experiencia previa es su guía. Pretende cruzar por Río Rico, Nogales, rodeando el cerro. Caminará durante cinco días para llegar a Tucson, Arizona, en donde trabajará para juntar dinero e ir a Phoenix, lugar en que sus patrones le darán trabajo. Lo que no nos contó es qué hará para esquivar a la mafia de Nogales, los "puntos», que monitorean constantemente el cruce del cerro para cobrar su obligado derecho de paso.

José Santos forma parte del 46 por ciento del grupo que encaja en la categoría de vulnerabilidad media. Este es el grupo más numeroso, un grupo casi exclusivo para varones con la excepción de una mexicana que con trabajos pudimos ubicar aquí, siendo que formaba finalmente parte de ese 32 por ciento de casos atípicos.

Vulnerabilidad alta o el factor de "ser mujer», "ser muy joven» o "muy viejo» (para el viaje)

El 35.5 por ciento del grupo de estudio responde a esta categoría, el segundo grupo más numeroso. Los tipos más recurrentes son mujeres con muy pocos recursos, migrantes varones muy jóvenes (entre 18 y 25 años) u otros migrantes de mediana edad (entre 40 y 57 ), muchos sin profesión y que realizan el viaje por primera vez. Ocasionalmente nos encontramos con menores de edad que viajaban acompañados por adultos desde el punto de origen. Este grupo viaja predominantemente en tren y de forma indocumentada, si bien su condición altamente vulnerable los empuja, en algunos casos, a utilizar el autobús en pequeños tramos. Aquí observamos que las redes de apoyo en el origen, destino o tránsito son muy escasas. Esta categoría se distingue de la anterior por la falta de experiencia previa de viaje, la ausencia de un oficio mínimamente consolidado y por el propio perfil del grupo (edad y sexo), el cual resulta menos favorable para acometer una aventura tan peligrosa. Como consecuencia de lo anterior, los que están en la categoría de vulnerabilidad alta, poseen notablemente menos recursos para afrontar las adversidades del viaje. En Estados Unidos suelen tener pocas redes (a veces 
ninguna), ya que nunca se establecieron ahí, quizás una tía, un familiar que apoya con algo de dinero. Muchas veces no saben a dónde llegarán en Estados Unidos, no tienen destino fijo y están «a lo que salga». Llama la atención la escasa planeación que hicieron del viaje. Si bien este tipo utiliza las estrategias de sobrevivencia del tipo anterior, su repertorio es más limitado (carece de experiencia propia que no suele ser suplida por redes de apoyo), aunque a veces, como veremos en el caso de las mujeres, más cuidado.

Podemos ilustrar esta categoría con el caso de Víctor, un salvadoreño de 22 años, sin oficio consolidado pero con cierta experiencia laboral, que viaja por primera vez a Estados Unidos. Viaja solo pero ha creado vínculos espontáneos durante el trayecto y viaja únicamente en tren a lo largo de la ruta del Pacífico. No conoce a nadie en Estados Unidos y su familia, en El Salvador, sólo lo apoya moralmente. Sabe que llegará a Tijuana, pero no sabe a dónde ir en Estados Unidos: «voy a ciegas», explica. No ahorró para hacer el viaje y sólo lleva 20 pesos encima. Ante la pregunta, ży cómo vas a seguir el camino sin dinero?, responde: «charoleo con mi grupito para comprar taco o gaseosa».

Como Víctor, encontramos predominantemente en esta categoría a jovencitos centroamericanos que viajan con lo puesto (si bien mayores de edad), personas mayores de cuarenta años o mujeres, todos con muy escasos recursos.

El grupo de mujeres que encontramos en esta categoría resulta digno de mención. Del total de mujeres entrevistadas, casi la mitad (46 por ciento) se ubica en vulnerabilidad alta. El grupo lo componen mujeres jóvenes, de un promedio de 29 años, en su mayoría amas de casa que viajan por primera vez a Estados Unidos. El 33 por ciento de ellas huye de situaciones de violencia intrafamiliar y 66.6 por ciento ha dejado hijos pequeños atrás. Otras viajan para reunirse con su pareja en Estados Unidos. Para este grupo el factor «familia» está muy presente en el motivo de la migración, además de la pobreza o falta de oportunidades. Salen para poder mandar dinero a sus hijos menores o para reunirse con la pareja que migró hace ya tiempo.

Ninguna ha estudiado más allá de primaria, todas hacen el viaje por primera vez, no han vivido en Estados Unidos y 66 por ciento dice no tener profesión. Esta falta de experiencia previa y de oficio, junto con el factor de 
"género», las hace especialmente vulnerables, aunque no tanto como las mujeres que encontramos en la categoría de vulnerabilidad extrema, en la cual se juntan otros factores aún más agravantes.

Vulnerabilidad extrema: menores no acompañados y mujeres en circunstancias agravantes

Si bien los grupos ubicados en vulnerabilidad alta se encuentran en condiciones muy adversas, no obstante existen situaciones aún peores. La vulnerabilidad extrema presenta características similares a la alta, pero con circunstancias agravantes. El factor de género y edad siguen siendo determinantes en esta categoría, si bien en ella encontramos migrantes que presentan circunstancias personales muy desfavorables: menores de edad que viajan sin acompañamiento y mujeres en condiciones especialmente difíciles; embarazadas, jovencitas que viajan con niños o menores a su cargo, mujeres agredidas durante el tránsito (entre otros casos). Estas circunstancias personales desfavorables, unidas al uso casi exclusivo de un medio de transporte especialmente peligroso (el tren), a la falta de experiencia previa del viaje, al nivel de violencia social e intrafamiliar que a menudo los golpea, la carencia de oficio y la precariedad de redes de apoyo, hace de éste el grupo más vulnerable de todos.

En este grupo observamos que los migrantes casi no tienen redes de apoyo en Estados Unidos o en su lugar de origen. Casi no planean su viaje y utilizan un repertorio menos surtido de estrategias de supervivencia, notablemente pedir dinero, formar grupos durante el viaje y, en ocasiones, ahorrar algo de dinero antes de salir o utilizar el autobús en algún pequeño tramo del sur de México. Incluso las mujeres que encontramos en esta categoría (a diferencia de las de vulnerabilidad alta) no manifestaron implementar estrategias especiales para protegerse durante el tránsito. La mejor fuente de información para este grupo parece ser el «boca a boca», la información que consiguen de otros migrantes (en sus países o durante el viaje), así como la que le proporcionan los albergues y casas de migrantes en México. En este sentido no acuden a fuentes adicionales de información.

Eva entra dentro de esta categoría extrema. Es una hondureña de 27 años, no tiene profesión y viaja exclusivamente en tren con cuatro hijos de entre 
4 y 7 años. Se dirige a Altar para trabajar en sembradíos de uva y pepino. Inició sola el viaje y en Arriaga conoció a un hombre, también en tránsito, con el que viaja hacia ese destino. Este acompañante esporádico no quiso ser entrevistado y alegó ser el padre de los niños, información que Eva negó posteriormente. Finalmente, nunca supimos qué papel desempeñaba en este pequeño grupo familiar en tránsito, pero los peores pensamientos nos asaltaron. Es la primera vez que Eva hace el viaje hacia el norte. Dice que emigra por razones de pobreza y violencia en su país. No lleva dinero encima, pero afirma que lo conseguirá pidiendo en vías públicas y trabajando.

Wilmer es un hondureño de 16 años que conocimos en Nogales. Cruzó México en tren, solo, sin conocer el país, pero logró hacer un grupo de amigos durante el viaje. Escapó de su casa sin informar a su madre (única familia que dice tener, junto con un hermano). Relata que en su país fue amenazado de muerte por grupos mareros al negarse a pertenecer a la pandilla. Viaja a Estados Unidos, pero dice no tener familia ahí (sólo unos conocidos en Nueva York, los cuales conoció en Honduras en el mercado en donde trabaja su madre) y no tiene un lugar de destino claro en ese país, ni en dónde, ni en qué trabajar. Su hermano en Comayagua, Honduras, le dio algo de dinero para el viaje y ha logrado sobrevivir haciendo uso de albergues y casas de migrantes. Un pequeño tramo del sur de México lo cubrió en autobús para evadir las zonas más peligrosas. Llegó hasta Nogales en tren e intentó cruzar la frontera en grupo, rodeando el muro, sin coyote y sin dinero. Relata que la mafia de Nogales los detuvo en el cerro, los amenazaron con pistola y los obligaron a continuar el cruce cargando una mochila repleta de mariguana. Wilmer logró escapar y volvió a Nogales, en donde obtuvo el apoyo y asesoría de la Iniciativa Kino. Se encuentra solo, sin dinero ni más apoyo que esta organización, donde le recomiendan que se entregue a las autoridades de migración de Estados Unidos y pida refugio por cuestiones humanitarias.

Wilmer es uno de los seis menores que entrevistamos para esta investigación. Todos se encontraban en circunstancias similares. Los niños centroamericanos del grupo de estudio (cinco de seis entrevistados) mencionaron — todos-que salieron de su país, además de por cuestiones de pobreza, por

$106 \frac{\text { PRIMER SEMESTRE } 2015}{\text { MIGRACIÓN Y DESARROLLO NÚM. } 24}$ 
problemas de violencia, principalmente la causada por el narcotráfico o los grupos pandilleros. Nos llamó la atención el hecho de que estos menores, en su mayoría (cuatro de seis), se dirigían a Estados Unidos sin tener familiares o conocidos en ese país, ni saber a dónde llegar o en qué trabajar. Con respecto a sus redes de apoyo en el país de origen, una mayoría manifestó tener familia que, en algunos casos, les apoyaron con un poco de dinero para hacer el viaje. Tres menores de seis mencionan que el padre no estaba presente en el núcleo familiar. Un menor menciona que fue abandonado por ambos padres. Estos datos reflejan la vida de niños que provienen de familias precarias y desmembradas. Están solos y son proclives a caer en redes de narcotráfico (usados como "burreros», como en el caso de Wilmer) o en el tráfico de personas. Son, como sugiere Vogt (2013), una mercancía con un alto valor de uso y cambio.

\section{Perfiles atípicos}

Dentro de la muestra, el 32 por ciento no responde plenamente a las categorías anteriores y presenta indicadores en varias de ellas. Los casos atípicos fueron ubicados finalmente en alguna de las categorías cuando la mayoría de los indicadores respondían a alguna de ellas, no obstante surgió la pregunta de quiénes integraban estos perfiles difíciles de clasificar. Observamos que muchos de ellos eran mujeres cuya condición de género hacía que se rompieran las categorías más comunes. Por ejemplo, mujeres que aunque presentan fuertes indicadores de vulnerabilidad alta (falta de experiencia previa en el viaje, no deportadas, sin oficio y que viajaban por primera vez), deciden hacer el viaje porque poseen redes densas de apoyo y, por su condición de mujer, son especialmente cuidadosas al planear y llevar a cabo el viaje (hacen uso de coyotes, viajan en autobús, duermen en hoteles, etcétera). En este sentido, las mujeres merecen una sección aparte y una explicación a partir de la mirada de género. Otros casos atípicos corresponden a hombres deportados de mediana edad, con desorientación y desarraigo en su retorno a México, sin rumbo claro por la geografía mexicana y sin planes claros para 
el futuro. Hombres que por su edad, dinámica de movimiento y circunstancias especiales, no responden a las categorías comunes pero que están dentro del flujo hacia el norte. También se inscriben, en este grupo, casos de migrantes aventureros con motivaciones distintas al migrante económico o mexicanos y mexicanas jornaleros que hacen un tramo del viaje y cuyas circunstancias son muy particulares. También jóvenes que, si bien presentan indicadores importantes en vulnerabilidad alta, caen en la media o moderada por los considerables recursos que poseen para el viaje (como fuertes redes de apoyo, mucha planeación y acceso a la información).

\section{FACTOR DE GÉNERO Y FACTOR DE NACIONALIDAD COMO CONDICIONANTES DEL VIAJE}

Como se demuestra en los apartados anteriores, la vulnerabilidad de los grupos estudiados está fuertemente determinada por factores como la edad, la experiencia previa del viaje, el oficio, las redes de apoyo, el género o la nacionalidad, no obstante estos últimos dos factores ameritan mayor análisis y explicación por resultar, en algunos casos, factores especialmente condicionantes.

Factor de género

Las mujeres entrevistadas representan 18.6 por ciento del grupo estudiado y muchas son migrantes independientes. La mayoría de ellas (69 por ciento) se ubica en las categorías de vulnerabilidad alta y extrema, lo que contrasta con los resultados obtenidos dentro del grupo de hombres adultos, en donde 33.3 por ciento se ubica dentro de la categoría de alta y ninguno en la de extrema.

De acuerdo con el grupo de estudio, la mujer presenta mayor vulnerabilidad que el hombre migrante, pero la mujer centroamericana tiene un perfil de vulnerabilidad aún más acusado: mientras que la mayoría de las mexica-

$108 \frac{\text { PRIMER SEMESTRE } 2015}{\text { MIGRACIÓN Y DESARROLLO NÚM. } 24}$ 
CUADRO 7

Categorías de vulnerabilidad diferenciada por sexo

\begin{tabular}{lcccc}
\hline & $\begin{array}{c}\text { VULNERABILIDAD } \\
\text { MODERADA }\end{array}$ & $\begin{array}{c}\text { VULNERABILIDAD } \\
\text { MEDIA }\end{array}$ & $\begin{array}{c}\text { VULNERABILIDAD } \\
\text { ALTA }\end{array}$ & $\begin{array}{c}\text { VULNERABILIDAD } \\
\text { EXTREMA }\end{array}$ \\
\hline $\begin{array}{l}\text { Mujeres } \\
\text { Hombres (adultos) }\end{array}$ & $23 \%$ & $7.6 \%$ & $46 \%$ & $23 \%$ \\
\hline
\end{tabular}

Fuente: Elaboración propia a partir del trabajo de campo de esta investigación.

nas se ubican dentro de la categoría moderada y media, todas las guatemaltecas y hondureñas encontradas clasifican dentro de las categorías de alta y extrema. No encontramos en el trabajo de campo mujeres salvadoreñas, dato para el cual no tenemos ninguna explicación.

Esta vulnerabilidad especial de la mujer indica que el factor de género tiene un peso crítico, dado el nivel de peligro que «ser mujer» tiene a la hora de hacer el viaje. ${ }^{6}$ Este abuso se relaciona, según Vogt, con la manera en que se ha construido la imagen de la mujer centroamericana en México: «las migrantes se han transformado en objetos de deseo sexual y la industria del sexo es altamente redituable, ya que las mujeres y los niños o niñas pueden venderse varias veces» (Vogt, 2013: 774). El cuerpo de la mujer migrante se ha mercantilizado y es objeto de abuso y lucro para distintos grupos. Si en México, en la construcción social que se hace de los hombres centroamericanos se les asocia con «violencia pandillera, delincuencia e introducción de vicios como el uso de drogas y alcohol», a las mujeres se les asocia con "prostitución e inmoralidad sexual, viéndolas como malas madres que dejan a sus hijos atrás» (Vogt, 2013: 770).

Frente a esta vulnerabilidad por razones de género la mujer migrante se distingue del varón en su forma de tomar decisiones y comportarse durante el viaje. Es más cuidadosa en la planeación del mismo y toma medidas pro-

${ }^{6}$ Se ha documentado ampliamente el abuso sexual contra la mujer migrante en México, así como los peligros de caer en redes de prostitución o trata de personas. Por ejemplo, véase el reporte de Amnistía Internacional (2010) o de la Comisión Nacional de los Derechos Humanos (2011). 
tectoras. Aunque muchas de estas mujeres se encuentran en el rango alto de vulnerabilidad y son de bajos recursos, no obstante optan por estrategias muy cuidadas (propias de la moderada o media) y viajan en autobús por los tramos más peligrosos, hacen uso de hoteles en ciertos puntos o incluso atraviesan México con coyote. En su recorrido en tren (cuando no hay más remedio), una estrategia recurrente es buscar la protección del grupo de hombres que han conocido por el camino.

\section{Factor de nacionalidad}

El factor de nacionalidad incide en el nivel de vulnerabilidad por el hecho de permitir a un grupo, el mexicano, viajar de forma documentada por el país. Sin embargo, no tenemos resultados conclusivos acerca de las posibles diferencias entre las nacionalidades centroamericanas y su relación con la vulnerabilidad. Esto se debe a que no logramos hacer el mismo número de entrevistas para todos los grupos analizados. Aunque salta a la vista que una mayoría de los mexicanos (y exclusivamente ellos) se ubican en la categoría moderada, lo cual podríamos explicar en función del tránsito regular que realizan por México y su mayor capital social específico en la migración, siendo que tienen tradiciones migratorias bien establecidas en Estados Unidos. Con todo, la vulnerabilidad sigue siendo elevada en este grupo, dado que 23 por ciento se ubica en la categoría de alta y 7.6 por ciento en extrema. Por contraste, el grupo de guatemaltecos, según estos resultados, es el más vulnerable de todos, siendo que 62.5 por ciento cae en las categorías alta y extrema. No encontramos una explicación para estos resultados, siendo que a menudo los hondureños son los considerados como más vulnerables en los reportes nacionales. Este grupo se muestra como el más numeroso dentro de la categoría media (58 por ciento) e incluso muestra los mayores niveles de estudio y de profesionalidad después de los salvadoreños.

Los salvadoreños se ubican en su mayoría en la vulnerabilidad media (53 por ciento) y un importante porcentaje (40 por ciento) está en alta. No obstante, resultan ser el grupo con el mayor grado de profesionalización

$110 \frac{\text { PRIMER SEMESTRE } 2015}{\text { MIGRACIÓN Y DESARROLLO NÚM. } 24}$ 
CUADRO 8

Comparativa de vulnerabilidad por nacionalidad en porcentajes

\begin{tabular}{lcccc}
\hline & $\begin{array}{c}\text { VULNERABILIDAD } \\
\text { MODERADA }\end{array}$ & $\begin{array}{c}\text { VULNERABILIDAD } \\
\text { MEDIA }\end{array}$ & $\begin{array}{c}\text { VULNERABILIDAD } \\
\text { ALTA }\end{array}$ & $\begin{array}{c}\text { VULNERABILIDAD } \\
\text { EXTREMA }\end{array}$ \\
\hline Hondureños & - & $58 \%$ & $35.4 \%$ & $6.4 \%$ \\
Guatemaltecos & - & $37.5 \%$ & $37.5 \%$ & $25 \%$ \\
Salvadoreños & - & $53 \%$ & $40 \%$ & $6.6 \%$ \\
Mexicanos & $53.8 \%$ & $15.3 \%$ & $23 \%$ & $7.6 \%$ \\
\hline
\end{tabular}

Fuente: Elaboración propia a partir del trabajo de campo de esta investigación.

(73.3 por ciento manifestaron tener un oficio consolidado) y educación (46.6 por ciento habían cursado algunos semestres de preparatoria). Posteriores investigaciones más centradas en la comparación de factores como educación, oficio y vulnerabilidad entre nacionalidades (sobre todo entre los grupos centroamericanos) podrían esclarecer muchas de las preguntas que resultan de estos hallazgos preliminares.

\section{CUADRO 9}

Comparativa de escolaridad y oficio por nacionalidad

\begin{tabular}{lcccc}
\hline & Sí TIENE PROFESIÓN & $\begin{array}{c}\text { PREPARATORIA } \\
\text { (FINALIZADA Y/O INICIADA) }\end{array}$ & $\begin{array}{c}\text { SECUNDARIA } \\
\text { (FINALIZADA Y/O INICIADA) }\end{array}$ & $\begin{array}{c}\text { PRIMARIA } \\
\text { (FINALIZADA Y/O INICIADA) }\end{array}$ \\
\hline Hondureños & $66.6 \%$ & $24.2 \%$ & $15 \%$ & $54.5 \%$ \\
Guatemaltecos & $56 \%$ & $6.25 \%$ & $50 \%$ & $37 \%$ \\
Salvadoreños & $73.3 \%$ & $46.6 \%$ & $26.6 \%$ & $26.6 \%$ \\
Mexicanos & $61.5 \%$ & $7.7 \%$ & - & $92.3 \%$ \\
\hline
\end{tabular}

Fuente: Elaboración propia a partir del trabajo de campo de esta investigación.

\section{Consideraciones finAles}

El concepto de vulnerabilidad utilizado en este trabajo se construye a partir de la relación entre los riesgos y la capacidad que tienen los migrantes para afrontarlos como consecuencia de una serie de recursos que convierten en 
estrategias de supervivencia. En este sentido, la perspectiva del actor social es enfatizada dentro del innegable contexto de constreñimientos estructurales. Las distintas categorías de vulnerabilidad que derivan del análisis de los grupos migratorios resultan de utilidad para lograr entender, con mayor profundidad y de manera más científica, las condiciones de agencia y desprotección de la población estudiada. Esto puede derivar en el establecimiento de mayores medidas de apoyo y cuidado para esta población en su tránsito por México.

Encontramos que la vulnerabilidad de los grupos estudiados está fuertemente permeada por factores como nacionalidad, género, edad, experiencia previa de viaje, redes de apoyo y condición socioeconómica. Los grupos menos vulnerables lo son básicamente por el factor de nacionalidad, donde sólo un grupo de mexicanos y mexicanas muestran relativamente mayores recursos para afrontar los peligros (el tránsito legal o la existencia de redes de apoyo más sólidas). Ciertas mujeres y niños, por su condición de género y edad, muestran una vulnerabilidad más alta y en ocasiones extrema. La yuxtaposición de ciertos factores dan como resultado la vulnerabilidad extrema, factores como ser mujer o ser menor que viaja solo, no poseer experiencia laboral o experiencia previa del viaje, falta de capital social, e incluso huir de situaciones de violencia social e intrafamiliar en los países de origen. En cambio, una mayoría del grupo de estudio, predominantemente masculina, califica en una vulnerabilidad media en donde los activos más importantes consisten en tener la edad ideal para el viaje, la condición masculina y la experiencia previa del tránsito por México y en el lugar de destino.

Si bien el grupo de estudio refleja mayoritariamente la existencia de grupos con escasos recursos y elevadísimos riesgos en el tránsito, no obstante los migrantes muestran capacidades para generar estrategias de supervivencia ante el peligro, haciendo uso de sus redes de apoyo en origen o destino, creando vínculos espontáneos con otros migrantes durante el viaje, planeando el itinerario y los medios de transporte, utilizando medios electrónicos o de telefonía, etcétera. Como es lógico, encontramos que a mayor cantidad y calidad de recursos, los migrantes son capaces de implementar más y mejores estrategias, lo que los coloca en un menor grado de vulnerabilidad relativa.

$112 \frac{\text { PRIMER SEMESTRE } 2015}{\text { MIGRACIÓN Y DESARROLLO NÚM. } 24}$ 
La ruta de occidente, actualmente considerada por los mismos migrantes como la más segura, podría ser una bomba de tiempo en el futuro, en el sentido de que a mayor flujo migratorio, mayor número de grupos criminales (clandestinos y de origen gubernamental o públicos con índices de corrupción preocupantes) tenderán a formarse alrededor de este tránsito. Si este flujo sigue incrementándose, sería de esperar que el nivel de abuso también lo haga, así como la inseguridad ciudadana en general. Asimismo, la política de contención migratoria implementada por Estados Unidos perjudica a México, dado que genera un «tapón humano» en la frontera que eventualmente se dispersa y recircula pero formando bolsas de estancamiento en puntos concretos del corredor. Esto genera problemas sociales y de seguridad en dichos puntos. Asumiendo este movimiento humano con mayores niveles de transparencia (saber quiénes son y en qué condiciones viajan) podemos «tempranamente» tomar medidas de protección y de desarticulación prematura de grupos criminales, a la vez que se cumple con la legislación internacional y, de ser el caso, devolver a las personas a sus países con la garantía de que éstos tomarán las medidas necesarias para crear condiciones favorables para su reinserción. En este sentido, necesitamos políticas migratorias regionales que atajen de raíz las causas perversas que generan la necesidad de migrar y, como muchos han dicho ya, cambiar el actual paradigma de seguridad nacional por otro de seguridad humana y económica.

\section{Bibliografía}

Amnistía Internacional (2010), Víctimas invisibles. Migrantes en movimiento en México, Londres, Amnistía Internacional.

Casillas R., Rodolfo (2008), «Las rutas de los centroamericanos por México, un ejercicio de caracterización, actores principales y complejidades», Migración y Desarrollo, vol. 6, núm. 10.

Castles, Stephen (2010), «Understanding Global Migration: A Social Transformation Perpective», Journal of Ethnic and Migration Studies, vol. 35, núm. 10.

Comisión Nacional de los Derechos Humanos (CNDH) (2009), Informe especial de la Comisión Nacional de los Derechos Humanos sobre los casos de secuestro en contra de migrantes, México, Comisión Nacional de los Derechos Humanos. 
(2011), Informe especial sobre secuestro de migrantes en México, México, Comisión Nacional de los Derechos Humanos. Disponible, en http://www.cndh. org.mx/sites/all/fuentes/documentos/Comunicados/2011/COM_2011_110. pdf (consultado el 23 de abril de 2012).

Chávez Galindo, Ana María y Antonio Landa Guevara (2012), «Migrantes en su paso por México: nuevas problemáticas, rutas, estrategias y redes», ponencia presentada en la XI Reunión Nacional de Investigación Demográfica en México, 30 de mayo-1 de junio, Aguascalientes, Aguascalientes, en http://www.somede.org/xireunion/ponencias/Migracion\%20internacional/147Pon\%20Ana\%20 Ma\%20Chavez-Antonio\%20Landa.pdf (consultado el 12 de junio de 2014).

Emif-Norte (2011), Encuesta sobre Migración en la Frontera Norte de México 2011, serie anualizada 2004 a 2011, México, Secretaría de Gobernación, Consejo Nacional de Población, Instituto Nacional de Migración, Unidad de Política MigratoriaCentro de Estudios Migratorios, Secretaría de Relaciones Exteriores, Secretaría del Trabajo y Previsión Social, El Colegio de la Frontera Norte, en http://www. colef.mx/emif/resultados/publicaciones/publicacionesnte/pubnte/EMIF\%20 NORTE\%202011.pdf (consultado el 15 de enero de 2015).

fm4 Paso Libre (2013), Migración en tránsito por la Zona Metropolitana de Guadalajara: actores, retos y perspectivas desde la experiencia de FM 4 Paso Libre, Guadalajara, Prometeo Editores.

FOOTE, Joanna y Mary Small (2013), Inseguridad permanente: abusos contra centroamericanos en México, Washington, Jesuit Refugee Service USA, en https://www. jrsusa.org/assets/Publications/File/Inseguridad_Permanente_web.pdf (consultado el 20 de junio de 2014).

IBARRA, Liliana (2009), «Diez años pasaron ya y sigo siendo un ilegal: la migración internacional como expresión de vulnerabilidad social», en Liliana Meza y Miriam Cuéllar (comps.), La vulnerabilidad de los grupos migrantes en México, México, Universidad Iberoamericana.

INEDIM (2011), «Seguridad para el Migrante: una agenda por construir», Documentos de Trabajo INEDIM, México, INEDIM.

MeYer, Maureen y Stephanie Brewer (2010), Un trayecto peligroso por México: violaciones a derechos humanos en contra de los migrantes en tránsito, Washington y México, Washington Office for Latin America y Centro de Derechos Humanos Miguel Agustín Pro.

Pérez de Armiño, Karlos (2009), «Vulnerabilidad y desastres. Causas estructurales y procesos de la crisis en Africa», Cuadernos de Trabajo de Hegoa, núm. 24, en

$114 \frac{\text { PRIMER SEMESTRE } 2015}{\text { MIGRACIÓN Y DESARROLLO NÚM. } 24}$ 
http://publ.hegoa.efaber.net/assets/pdfs/123/Cuaderno_de_trabajo_24.pdf? 1304002061 (consultado el 30 de noviembre de 2013).

RIVAS Castillo, Jaime (2011), «ỉVíctimas nada más? Migrantes centroamericanos en el Soconusco, Chiapas», Nueva Antropología, vol. xxiv, núm. 74.

Rocha, Jorge, Paola Escalona y Fernando Gutiérrez (2011), Migración centroamericana en su paso por la zona metropolitana de Guadalajara, Guadalajara, Programa Institucional de Derechos Humanos y Paz del ITESo y fm4 Paso Libre Dignidad y Justicia en el Camino.

Secretaría de Gobernación (Segob), Boletines estadísticos, México, Unidad de Política Migratoria, en http://www.gobernacion.gob.mx/es_mx/SEGOB/Boletines_ Estadisticos (consultados el 2 de febrero de 2015).

Southwest Border Sectors (2014), "Aprehensions by Fiscal Year», en www.cbp/gov/ sites/default/files/documents/u.s.\%20Border (consultado el 20 de mayo de 2015).

VoGt, Wendy (2013), "Crossing Mexico: Structural Violence and the Commodification of Undocumented Central American Migrants", American Ethnologist, vol. 40, núm. 4, en http://www.academia.edu/5073265/Crossing_Mexico_Structural_ violence_and_the_commodification_of_undocumented_Central_American_ migrants. Consultado 9/7/14) (Consultado el 20 de abril de 2014).

\section{ENTREVISTAS}

Machuca, Ricardo (2014), Director de programas en México para la Iniciativa Kino, Nogales, Sonora, 28 de febrero.

Martínez, Iliana (2014), Profesora-investigadora del Programa de Migración del rTeso, Guadalajara, 12 de marzo. 
